\title{
Space Photography and the Exploration of Mars
}

\author{
B. C. Murray and M. E. Davies
}

\begin{abstract}
A general exposition of the scientific potentialities and analytic framework of space photography is presented using the photography of Mars from flybys and orbiters as the principal example. Space photography is treated here as a communication process in which planetary scene information is communicated to the eye-brain receiver of earth-based interpreters. The salient parameters of this process are: (1) total information returned, (2) surface resolution, and (3) a priori knowledge regarding the planetary surface observed.
\end{abstract}

\section{Introduction}

With the advent of the telescope, Mars became competitive with the moon and sun as the focus of man's interest beyond his own world. The discovery of the white polar caps, their migration from one pole to the other, and the darkening of certain surface features led to the widespread notion that Mars was an earth-like planet. In addition, the remarkable similarity of the rotation periods and obliquities of the two planets further enhanced the comparison. Then the controversy concerning the nature of the "canals," thought by Lowell to be evidence of intelligent civilization, impressed Mars on man's consciousness with an intensity still apparent half a century later in the public response to the return of close-up Mars photography from spacecraft.

As indicated in Fig. 1 and Table I, we are in the midst of an information revolution concerning Mars; this is especially true of photographic information. Major changes of scientific opinion about Mars have already taken place, particularly concerning the supposed similarity to earth, and much more change is surely at hand. Thus, it is appropriate at this time to review some of the technical basis of Mars photography from flybys and orbiters, emphasizing especially those aspects of space photography specific to Mars which have been developed so far. However, there is a broader significance: the techniques developed and the surprises encountered in the exploration of Mars represent the background and experience for the photographic exploration of all the other planets, a historic process which will add meaning and significance to man's next

B. C. Murray is with the Division of Geological Sciences, California Institute of Technology, Pasadena, California 91109; M. E. Davies is with The Rand Corporation, Santa Monica, California.

Received 19 December 1969. decade just as the exploration of the earth and moon by space photography has enriched the past decade.

The photographic exploration of Mars, and that of all temporally varying planets, is a multivariable process in which surface resolution, geographic coverage, and observation time are all of importance. Thus, the conception and design of photographic missions is concerned with the balancing of these and other factors against the total photographic data that can be returned from the spacecraft. In this paper we will emphasize photography as a communication process in which information flows from the object being studied to the minds of scientists on the ground. We attempt to place this process in a quantitative framework in Secs. III-VIII, drawing examples from the exploration of Mars but indicating applications elsewhere as well. The concluding Sec. IX is concerned with how best to proceed further with the photographic exploration of Mars as well as the question of balance between the exploration of Mars and the rest of the solar system.

Before proceeding into the technical analyses of Secs. III-VIII, however, it is important to discuss the scien-

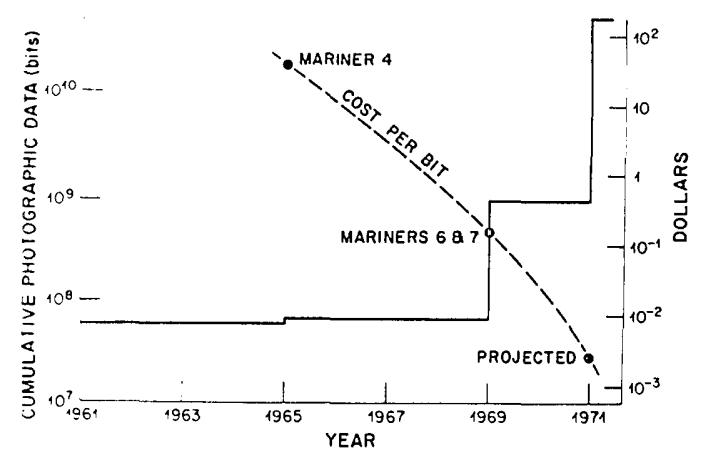

Fig. 1. Photographic exploration of Mars. The accumulation of photographic data (in bits) is shown as a function of time as is the sharply decreasing cost/bit. Data from Table I. 
Table I. Photographic Exploration of Mars

\begin{tabular}{|c|c|c|c|c|}
\hline & $\begin{array}{c}\text { Total data return } \\
\text { (bits) }\end{array}$ & Observation time & $\begin{array}{l}\text { Best surface } \\
\text { resolution } \\
(\mathrm{km})\end{array}$ & Total cost $(\$)^{c}$ \\
\hline $\begin{array}{l}\text { All ground-based obser- } \\
\text { vations } \\
\text { (telescopic) }\end{array}$ & $7 \times 10^{7} ?^{a}$ & $100 \%^{b}$ & 100 & $?$ \\
\hline $\begin{array}{l}\text { Mariner } 4(1965) \\
\quad \text { (flyby) }\end{array}$ & $3.5 \times 10^{6}$ & negligible & 3 & $1.25 \times 10^{8}$ \\
\hline $\begin{array}{l}\text { Mariners } 6 \text { and } 7 \text { (1969) } \\
\text { (flyby) }\end{array}$ & $5 \times 10^{8}$ & $1 \%$ & 0.3 & $1.5 \times 10^{8}$ \\
\hline $\begin{array}{l}\text { Mariner Mars }{ }^{\prime} 71^{c} \\
\quad \text { (orbiters) }\end{array}$ & $5 \times 10^{10}$ & $16 \%$ & 0.15 & $1.5 \times 10^{8}$ \\
\hline
\end{tabular}
Note: All data are approximate.
a Estimated from Ref. 9.
${ }^{b}$ Not consecutive coverage in time.
$c$ Projected data based on current plans.
${ }^{d}$ Assuming entire mission cost is to be borne by photography even though other instruments are also carried.

tific value of this enterprise. Accordingly, Sec. II presents a discussion of the scientific utility of Mars photography which reflects some of the attitudes and experiences of the Mariner 6 and 7 television team, of which both authors are members.

\section{Science, Exploration, and Space Photography}

Perhaps the most important single justification for space photography is its exploratory potential. More than any other kind of observation, a picture can reveal an aspect or condition of the planet which was not even imagined. The discovery by Luna 3 that the hidden hemisphere of the moon exhibited practically no maria, and the finding by Mariner 4 that the surface of Mars closely resembled that of the moon, are illustrations of space photography ventures in which the principal results were never specifically considered by the experimenters beforehand. On the other hand, a picture is not limited in its usefulness simply to the unexpected things it might reveal. On the contrary, a surprisingly long list of important specific scientific objectives of space photography can be formulated. Thus any particular mission must develop a strategy for pursuing such objectives without compromising the capability for exploratory discovery.

In the case of Mars, both fixed and variable features exist and scientific objectives are easily grouped into corresponding categories. There are three principal aspects of the fixed Martian features for photographic investigation: (1) tectonic classification; (2) crater statistics and morphology, and (3) local surface environments.

Tectonics-the study of patterns and processes of deformation - affords one approach to what is perhaps the most outstanding geological question concerning Mars: to what extent is it a close relative of the moon in terms of internal structure and, especially, chemical history? Did it ever form an extensive atmosphere in which the initial development of life could have taken place, or has it always resembled the moon as much as it does now? Mountain building deformation is generally regarded as a sensitive indicator of chemical differentiation on a planetary scale. Hence, the existence of large scale topographic features of internal origin is a most important surface clue as to internal development. High priority therefore has been given in the photographic exploration of Mars to the search for remnants of arcuate mountain systems, folded mountains, and, generally, endogenic features unlike those of the moon.

As indicated first by Mariner 4, the surface of Mars is generally cratered like that of the moon but is much smoother. Mariners 6 and 7 confirm this general appearance for other areas of the planet but also have discovered that the bright desert Hellas is an extraordinary featureless plain, and there are other nonlunar terrains, implying the existence of geographic variations in surface processes and/or materials. Extension of such photography in 1971 over a large fraction of the planet, and down to about $1-\mathrm{km}$ resolution, as well as selected high resolution sampling down to around 100-m resolution, should permit mapping of what are evidently a considerable variety of morphological provinces on Mars, and thus provide the basis for unraveling the major elements of Mars history and surface processes. The question of the weathering, transportation, and sedimentational processes responsible for the greater smoothness of Mars compared with the moon is being attacked with the Mariner 6 and 7 photography by intercomparison of areas on the planet at different latitudes and with different geomorphological associations, as well as analysis of the variety of detail on and about the south polar cap.

Perhaps the most important practical question regarding the search for life on Mars is where to land. Most specialists expect that life, if it exists at all, will be highly concentrated in favorable surface environments, perhaps constituting only a tiny fraction of the 
over-all planetary surface area. Mars exhibits a somewhat broader range of local environments than cloes the moon, but the range must be quite restricted compared with that of the earth. Thus the search, at high resolution, for characteristic differences between local areas, and the inferential identification of distinct classes of local surface environments should be of high priority. This same procedure is also a most desirable way to investigate the question of the nature of the erosional processes on the planet.

The variable features of Mars can be grouped into four categories for consideration of the role of photography in their study: (1) clouds and transient frost deposits; (2) the polar caps and other long-duration frosts; (3) the so-called "blue haze" and other obscurations; and (4) the wave of darkening.

Although the first analyses of the Mariner 6 and 7 close-up pictures have failed to yield a single positively identified cloud, the potential significance of $\mathrm{TV}$ monitoring of Martian clouds and frost deposits from an orbiter, in contrast to a flyby, is very great. Even occasional photographic observation of clouds, for example, can indicate much about atmospheric structure in the cloudy region: the processes forming the cloud, such as radiative cooling, orographic lifting, or thermal convection; the thermal structure of the atmosphere (e.g., stable or unstable); and possiblyand of great significance-the local atmospheric moisture content. Such observations would be of great importance in indicating the characteristics of local or temporary surface environments. If clouds are comparatively frequent, as may be the case in the " $W$ " cloud area observed in the far encounter phase of the Mariner 6 and 7 photography, the extended observational capability of an orbiter immediately provides advantages. It might then be possible to determine characteristics of the moisture climatology from cloud and haze distributions. Cloud cover could probably be related to small or large scale topographic features. Perhaps most significantly, wind patterns and their time variations could be mapped across portions of the planet. One special significance of transient frost deposits is that they may provide clues to localities where water vapor is passing into or out of the soil and, hence, to the possible temporary existence of soil moisture.

Depending on orbital and camera mechanization choices, changes in shape of the edge and interior of the polar cap could be observed in 1971, a subject of special interest after the first look provided by Mariner 7 . Additional data on slopes of the underlying ground may be available from photometric analysis of the uncovered terrain photographs.

Ground-based observers have for many years referred to an absorbing blue haze on Mars, although some workers argued the phenomenon was a surface effect. Surface topography is clearly visible in the blue filtered pictures of Mariner 6 and 7, thus removing another myth about the atmosphere of Mars. Conversely, it can be argued that the risking of the $25 \%$ of the wide angle frames taken with a blue filter paid off in the 1969 flights, thus permitting the 1971 mission to operate more effectively.

The 1971 mission will provide the first opportunity to study the wave of darkening from the neighborhood of Mars, although the 1969 mission has sampled the topography in light and dark areas and may have collected significant clues. This phenomenon consists of a seasonal change in contrast between the bright and dark areas which proceeds from the sublimating springtime polar cap toward and past the equator. The borders of the maria appear to become sharper, the maria darken relative to the deserts, and, according to some observers, they may develop blue or green colorations. The effect fades with the advances of the seasons and is followed by a second wave from the opposite pole half a Martian year later.

It was the wave of darkening that first suggested to astronomers that Mars might have vegetation. According to this view, the effect is caused by growth of vegetation in response to the seasonal availability of water vapor. Other, nonbiological, causes have also been proposed. These include wind-blown volcanic ash, atmospheric haze, physical changes in the soil due to freezing and thawing, the hydration of hygroscopic salts, etc. No theory has won general support, and the wave of darkening remains the major mystery of the planet. Photographic observations of interest to be repeated from an orbiter include: the relation of light and dark areas to the local topography, the fine structure of the dark regions, the existence of topographic or other differences between permanently dark areas and those that show large seasonal variations, the question of color changes in the dark regions, and observations on the rate and direction of the wave at a higher resolution than has yet been possible. The value of these studies will be enhanced by combining them with measurements of the local temperature and water vapor concentration.

Another important scientific aspect of space photography is that it provides the basis for high resolution maps. Topographic maps are needed for both the fixed feature studies such as geology and as a backdrop for the variable feature studies, as the relationship between the topography and surficial change can be very significant. Frequently other remote sensing experiments depend on accurate maps and sometimes pictures themselves to permit interpretation of their data. The Mariner 6 and 7 photography was carefully designed so that whole disk photography (far encounter) could be combined with close-up photography (near encounter) to produce useful maps. On the other hand, Mariner television cameras, as well as the lunar orbiter film systems, were not designed with mapping as a major 
objective; thus, there are many instrumental difficulties not normally encountered in terrestrial mapping. ${ }^{*}$

Even on the basis of the brief discussion above, it is clear that space photography of Mars contains enormous scientific potential. Clearly, the design and execution of that photography must constantly reflect interest in and knowledge of its diverse scientific uses if that potential is to be fully realized. The enormous lay interest in close-up photography of Mars should not obscure the fact that such photography constitutes a valid scientific investigation of paramount importance.

\section{What is a Photograph?}

A photograph is an intermediate recording and filtering of the brightness distribution in the scene viewed by the camera. When that representation of the visual scene is, in turn, viewed and interpreted by the human eye-brain combination, then and only then does a photograph have meaning. A photograph thus can be regarded as nothing more or less than a link in a communication system. This is a particularly appropriate basis on which to consider planetary photography from space probes because the intrinsic difficulty and expense of returning photographic information from planetary distances demand a most careful review of what signals really need to be transmitted by the communication system. What is it we wish to know about the visual scene as viewed from space? How can that information be most effectively recorded and returned to the ground? How can the view then be reconstructed to relay to the human observer all the information actually transmitted? These are the basic questions that have confronted those who have photographed the moon and Mars from space.

Not only is a photograph a representation of the scene viewed by the camera, it is a record on some sensor of the actual light intensity distribution in the focal plane of the camera. The imaging process itself involves some distortion of the incident radiation; uniform plane waves collected by the optical system will be imaged on the sensor as some small, but finite, bundle of light rays, a process described by the notion of a point spread function. Thus, the photographic record of the scene intensity distribution is one in which the fine detail has been smeared out somewhat by the point spread function of the optical system itself. However, the recording by the sensor of that image intensity distribution contributes further blurring. Thus, the eventual output of the sensor, be it voltage,

\footnotetext{
* The orbiter readout system mechanically segmented the frames in a very awkward manner making reassembly difficult, especially for mapping uses. The Mariner 6 and 7 television cameras not only suffer from the large distortions and residual image characteristic of slow-scan vidicons, but display a heretofore undetected dependence of apparent position on intensity. This new distortion, while only perhaps a $1 \%$ effect, further complicates the geometric reduction of the Mariner 6 and 7 imagery.
}

transmissivity, or other physical quantity, will exhibit the combined blurring of both the optical system and the sensor. Such angular effects are important to the question of resolution, which will be discussed later.

It is also important to consider the sensor as a light intensity transducer and to consider its signal and noise characteristics. Any small area of its surface can be considered to have some responsivity $\rho$, which relates the output physical quantity $V$ to the incident light intensity in the image plane $i$. Hence, the signal output will be $V_{S}=\rho \times i \times a$, where $a$ is the reference small area of the sensor involved.

The equivalent noise input, $\delta i$, on the other hand, may have some (perhaps complex) dependence on both the absolute area of the sensor involved and on the incident intensity. Hence, we can state, functionally at least,

$$
\delta i=f(i, a)
$$

and

$$
r_{N}=\rho \times \delta i \times a,
$$

where $V_{N}$ is in units of equivalent signal output. Since $\rho$ presumably is known from calibration data, we can alternatively speak of $i$ and $\delta i$, the reconstructed image intensity and its associated noise. The output signalto-noise is simply $V_{S} / V_{N}$. In space photography there are generally a number of extra links in the communication chain associated with returning the data to earth, and noise may creep in along the way. Therefore, we include in $\delta i$ all forms of noise arising between the original image plane and the finally reconstructed view on the earth and reference them in terms of equivalent input intensity.

Thus a generalized photographic system may be regarded as a remote viewing system for an earthbased observer, generally with time delay, in which: (1) the scene viewed undergoes angular blurring arising from the camera optics and the sensor; (2) the light intensity corresponding to each small element in the viewed scene is transduced into some measurable physical quantity and is ultimately displayed as a related shade of gray; and (3) spurious variations in gray shades, i.e., noise, are also present.

\section{Bits and Pieces}

If any photograph is examined at progressively increasing magnification, the variations of gray level within the area under observation will be found to become indistinguishable as the area is made smaller and smaller. We denote tentatively an individual small area within which only a single intensity can be discerned as a pixel (shorthand for picture element); a photograph can be regarded as composed of a large number of adjacent pixels. Later we will refine the notion of a pixel. If each pixel is taken to be $1 / K \mathrm{~mm}$ in size, and the photograph is $A \mathrm{~mm} \times B \mathrm{~mm}$ in dimension, then the number of pixels in the picture $n_{p}$ is

$$
n_{p}=K^{2} A B \text {. }
$$

However, the intensity corresponding to an individual 
pixel $(m, n)$ can be determined only with some limited accuracy due to the noise from the original sensor, or from subsequent steps in the communication process. Now at this point we wish to tie our analysis directly to the notions of communications by introducing the concept of a bit, the unit of binary encoding. Bits can be used as units of measure of the combined number of pixels and gray shades in a photograph. They provide a most suitable reference as to the communications requirement of a photograph.

Specifically, we wish to know how many zero-or-one characters in a binary word would be required to encode the sensor output $V_{m n}$ for transmission, i.e., how many distinct levels will be available to encode the output of each pixel. There is, of course, no exact way to answer such a question, since the more precisely $V_{m n}$ is encoded, the more accurately, in principle, the original intensity $i_{m n}$ can be inferred in the presence of the uncertainty $\delta i_{m n}$. But we note that, in reality, precision of encoding beyond a certain point helps mainly to provide improved knowledge of $\delta i_{m n}$, and only indirectly of $i_{m n}$. Thus efficient encoding practice often is based on the notion that the uncertainty in $i_{m n}$ resulting from the limitations of encoding should be roughly comparable to the intrinsic uncertainty in $i_{m n}$ itself, $\delta i_{m n}$.

Hence we wish to relate the signal-to-noise per pixel to the number of levels per binary word.

In this case $n_{b}$, the number of bits in each binary word, is related to the signal-to-noise by

$$
2^{n_{b}}=1+(i / \delta i)
$$

or the number of bits/pixel, $n_{m n}$, is

$$
n_{m n}=3 \log _{10}[1+(i / \delta i)] .
$$

This must be viewed as a very simplified approach to estimation of bit requirements of a photograph. Yet it does permit us to estimate the number of bits in a photograph with about the same accuracy as one can refer to the number of gray levels present. Thus the total number of bits in a given photograph, $n_{T}$, can be stated approximately as

$$
n_{T}=n_{p} n_{m n}=3 K^{2} A B \log _{10}[1+(i / \delta i)] .
$$

\section{Why Wander into the Space Frequency Domain?}

In many observational systems it has been found useful to analyze the temporal frequency content of the output signal. An analogous situation exists for photographic systems concerning the frequency distribution of the spatial variations in intensity which constitute the original and reconstructed images. There is, of course, only very rarely any physical significance to a particular space frequency in a scene. However, white light photographic systems (temporally incoherent) are reasonably linear in intensity in the sense that the intensity distribution in the image plane is the arithmetic sum of the intensity distribution arising from each small element of the quasi-random (spatially incoherent) intensity distribution of the scene being viewed. Under such conditions, the image degradation of any scene, in principle, can be accurately predicted from knowledge of the point spread function of the optical system. However, the actual usefulness of the point spread function is limited due to practical computational difficulties; instead the Fourier transformed functions, which are separable, have proven to be the more important. For example, the line spread function for each of a number of optical elements can be considered together as $1\left(x^{\prime}\right){ }^{*}$ Denoting the original intensity distribution in the scene being viewed as $o(x)$ and that at the corresponding point in the image plane as $i\left(x^{\prime}\right)$, then $\dagger$

$$
i\left(x^{\prime}\right)=\int_{-\infty}^{\infty} 1\left(x^{\prime}-x\right) o(x) d x .
$$

By comparison, if $\omega$ denotes spatial frequency, the cumbersome term $1\left(x^{\prime}\right)$ can be represented instead as a space frequency filter function $T(\omega)$,

$$
T(\omega)=\int_{-\infty}^{\infty} 1\left(x^{\prime}-x\right) \cos \omega x d x .
$$

Then

$$
1(\omega)=T(\omega) 0(\omega)
$$

and

$$
i\left(x^{\prime}\right)=\int_{-\infty}^{\infty} T(\omega) 0(\omega) \cos \omega x d \omega,
$$

where the capital letters signify the Fourier cosine transforms of the lower case intensity functions. The usefulness of the space frequency approach is obvious when it is recognized that if sinusoidal (or even square wave) bar charts are used to investigate optical performance, then the observed contrast reduction of the image compared to the target is a direct measure of the attenuation of the space frequency in question:

$$
T(\omega)=M^{\prime}(\omega) / M(\omega),
$$

where $M$ is the modulation and is defined as $i_{\max }-$ $i_{\min } / i_{\max }+i_{\min }$. The prime refers to the image plane observation. $T(\omega)$ is known as the modulation transfer function (MTF) and can be used to describe the angular blurring properties of the sensor. However, sensors normally are not completely linear over the entire intensity ranges involved, and it must be presumed that the output signal $V_{m n}$ has already been converted back to the apparent image intensity $i_{m n}$ by means of the known calibration $\rho(m, n)$ before the contrast reductions are considered. $\ddagger$ Thus, if we denote the MTF of the optics as $T_{0}(\omega)$, then

$$
1(\omega)=T_{0}(\omega) T_{s}(\omega) 0(\omega) .
$$

\footnotetext{
* Assuming a symmetrical line spread function.

$\dagger$ Somewhat simplified from the one-dimensional derivation of Smith (Ref. 1, p. 339).

† See "effective image" concept discussed by Brock (Ref. 2, p. $45)$
} 
Table II. Mariner Mars Television Cameras

\begin{tabular}{|c|c|c|c|c|c|}
\hline & \multirow[b]{2}{*}{ Mariner 4 (1965) } & \multicolumn{2}{|c|}{ Mariners 6 \& 7 (1969) } & \multicolumn{2}{|c|}{1971 Orbiter } \\
\hline & & Camera A & Camera B & Camera A & Camera B \\
\hline \multicolumn{6}{|l|}{ Optics } \\
\hline Type & 2-mirror cassegrain & 6 -element lens & $\begin{array}{l}\text { 3-element cata- } \\
\text { dioptric }\end{array}$ & 6-element lens & $\begin{array}{l}\text { 3-element ca- } \\
\text { tadioptric }\end{array}$ \\
\hline Focal length & $30.5 \mathrm{~cm}$ & $5 \mathrm{~cm}$ & $50.8 \mathrm{~cm}$ & $5 \mathrm{~cm}$ & $50.8 \mathrm{~cm}$ \\
\hline $\begin{array}{c}f / \text { no. } \\
\text { Shutter }\end{array}$ & $f / 8$ & $f / 5.6$ & $f / 2.5$ & $f / 4$ & $f / 2.5$ \\
\hline Type & intermittent rotary & intermittent rotary & $\begin{array}{l}\text { twin solenoid } \\
\text { two blade }\end{array}$ & $\begin{array}{l}\text { twin solenoid } \\
\text { two blade }\end{array}$ & $\begin{array}{l}\text { twin solenoid } \\
\text { two blade }\end{array}$ \\
\hline Exposure range & $0.08-0.20 \mathrm{sec}$ & $0.06-0.12 \mathrm{sec}$ & $\begin{array}{l}0.006-0.012 \\
\text { sec }\end{array}$ & $\begin{array}{l}0.024-0.192 \\
\quad \text { sec }\end{array}$ & $\begin{array}{l}0.006-0.048 \\
\text { sec }\end{array}$ \\
\hline Filters & green, orange & green, orange, blue & minus blue & $\begin{array}{l}\text { uv, blue, green, } \\
\text { orange, } 3 \\
\text { polarizers, } \\
\text { minus blue }\end{array}$ & minus blue \\
\hline \multicolumn{6}{|l|}{ Sensor } \\
\hline Type & slow scan vidicon & slow scan vidicon & $\begin{array}{l}\text { slow scan } \\
\text { vidicon }\end{array}$ & $\begin{array}{r}\text { slow scan } \\
\text { vidicon }\end{array}$ & $\begin{array}{r}\text { slow scan } \\
\text { vidicon }\end{array}$ \\
\hline $\begin{array}{l}\text { Format } \\
(\mathrm{mm})\end{array}$ & $5.6 \times 5.6$ & $9.6 \times 12.5$ & $9.6 \times 12.5$ & $9.6 \times 12.5$ & $9.6 \times 12.5$ \\
\hline $\begin{array}{l}\text { Format } \\
\quad \text { (pixels) }\end{array}$ & $200 \times 200$ & $704 \times 945$ & $704 \times 945$ & $700 \times 832$ & $700 \times 832$ \\
\hline $\begin{array}{l}\text { Field of view } \\
\quad \text { (degrees) }\end{array}$ & $1.05 \times 1.05$ & $11 \times 14$ & $1.1 \times 1.4$ & $11 \times 14$ & $1.1 \times 1.4$ \\
\hline $\begin{array}{l}\text { Frame time } \\
\quad(\mathrm{sec})\end{array}$ & 24 & 42 & 42 & 84 & 84 \\
\hline \multicolumn{6}{|l|}{ Storage } \\
\hline Type & $\begin{array}{l}\text { magnetic tape } \\
\text { digital }\end{array}$ & \multicolumn{2}{|c|}{$\begin{array}{l}\text { magnetic tape mixed analog } \\
\text { and digital }\end{array}$} & \multicolumn{2}{|c|}{ magnetic tape digital } \\
\hline$A / D$ encoding & 6 bits & \multicolumn{2}{|c|}{8 bits } & \multicolumn{2}{|c|}{9 bits } \\
\hline Total storage & $5 \times 10^{6}$ bits & \multicolumn{2}{|c|}{$1.8 \times 10^{8} \mathrm{bits}$} & \multicolumn{2}{|c|}{$1.8 \times 10^{8} \mathrm{bits}$} \\
\hline
\end{tabular}

Figure 2 shows MTF's appropriate for some photographic systems used in lunar and planetary exploration, and Table II supplies basic information on the Mars camera systems.

Thus the recourse to the space frequency domain is a useful way to measure the angular blurring effects of any photographic system. Equally important, it offers a way to correct for at least some of these effects. Equation (12) is separable. Therefore,

$$
0(\omega)=1(\omega) / T_{0}(\omega) T_{s}(\omega)
$$

and*

$$
o(x)=\int_{-\infty}^{\infty} 0(\omega) \cos \omega x d \omega .
$$

In principle at least, the image intensity distribution $l\left(x^{\prime}\right)$ can be transformed to the space frequency domain, the resulting coefficients $l(\omega)$ increased by $\left[T_{0}(\omega) T_{s}\right.$

\footnotetext{
* We continue to use only the one-dimensional cosine transform for illustration. Complete solutions generally require twodimensional formulation of both sine and cosine transforms, although this complexity is not required for all applications.
}

$(\omega)]^{-1}$ to yield estimates of $0(\omega)$, and that result transformed back to yield an estimate of $o(x)$. The limitation to such a process ultimately will be the sensor or other image noise, $\delta i(x)$, which will also be increased by

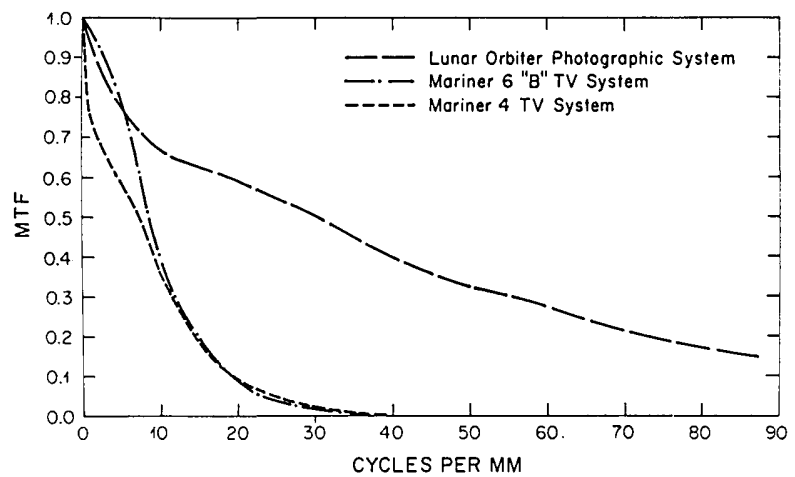

Fig. 2. Modulation transfer curves. The MTF curves for the vidicon camera systems of the Mariner missions to Mars in $1965^{3}$ and in 1969 (unpublished preliminary data) are compared with that of the Lunar Orbiter film/readout camera system ${ }^{4}$ flown in 1966. Horizontal and vertical MTF's have been averaged for the vidicon systems. 


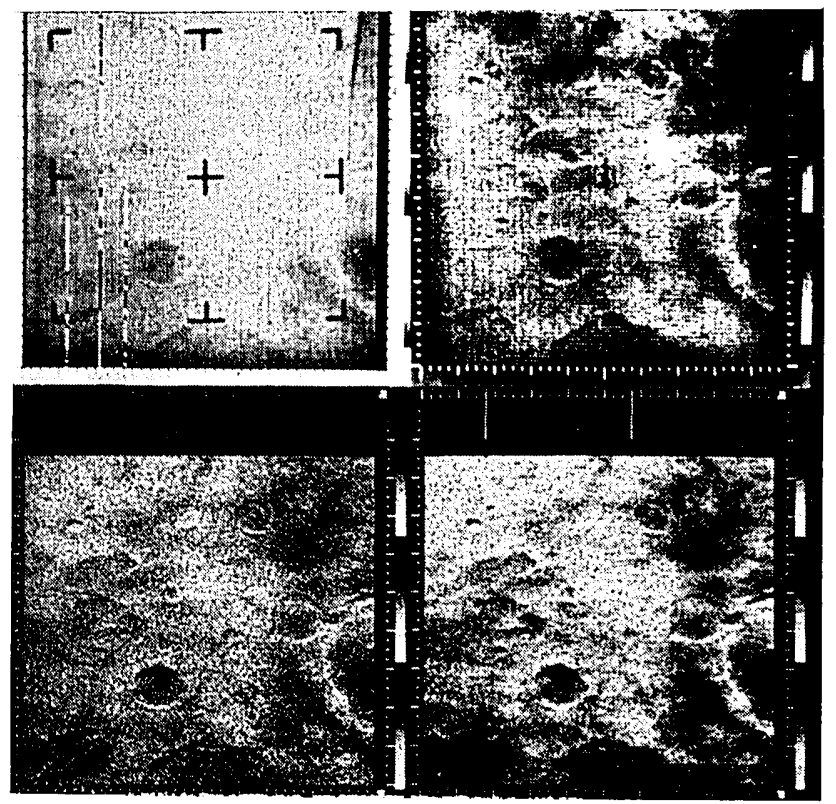

Fig. 3. Processing of Mariner 4 television picture. Upper left: raw data of frame 10 from Mariner 4 as received in July 1965. Upper right: same data, after several days of processing with preliminary enhancement and with torn lines corrected and fiducial marks and spurious interference removed by interpolation. Lower right: finally calibrated and enhanced photograph with most electrical noise removed. Lower left: same, except processed with high space frequency filter. The last two steps were not completed until more than a year after receipt of data. Details of processing are found in Ref. 3 .

$\left[T_{0}(\omega) T_{s}(\omega)\right]^{-1}$ and ultimately will make the reconstruction process unmanageable. Successful application of such filtering to improve resolution has been carried out extensively with lunar and Martian television photographs where (1) the signal-to-noise for each pixel was generally high; (2) the data were already in a form well suited for processing by digital computers; and (3) electrical interference also needed to be removed. More extensive use of filtering in the reconstruction of TV pictures can be expected as the techniques for doing so become better known.

Up to this point, our interest in filtering has been merely to help reproduce the original scene as faithfully as possible. However, the eye-brain combination can sometimes extract more information from the photograph if the contrast change in the reconstructed image has been artificially enhanced,i.e., by use of high gamma processing procedure with film. Similarly, it is sometimes desirable artificially to sharpen the detail in the picture at the expense of the low space frequency information. Both kinds of extreme enhancement have been carried out with the Mariner 4, 6, and $7 \mathrm{TV}$ pictures of Mars in order to convert the precise intensity data into information about the extremely low contrast scenes, as illustrated in Figs. 3 and 4.

These examples again lead us to the basic connection between angular resolution and the signal-to-noise of individual resolution elements. This, indeed, is one of two unifying concepts underlying the whole complicated process of design and utilization of photographic systems in space. With it, the system parameters can be adjusted favorably to maximize the useful pictorial data transmitted back from space.

\section{Resolution and Contrast}

After the space photographer has made sure he is communicating back from space all the bits he can justify, he then must wrestle with the question of how to expend them most effectively. Should he go for a few isolated very high-resolution pictures, or lots of contiguous low resolution coverage, or, as usually is best, some mixture of these extremes? Thus a clear understanding of the concept of resolution is necessary. In the testing of optical systems, resolution is a measure of the ability of the system to render barely distinguishable, to a human observer, a standard pattern of black and white lines.

However, the resolution defined in this way obviously depends on the contrast of the bar chart. If the spaces between the lines are only twice as bright as are the bars, i.e., a contrast of $2: 1$, then the barely detectable line spacing will be coarser than if the contrast were 100:1. Hence the limiting resolution of photographic systems often is stated as a certain number of lines per milli-

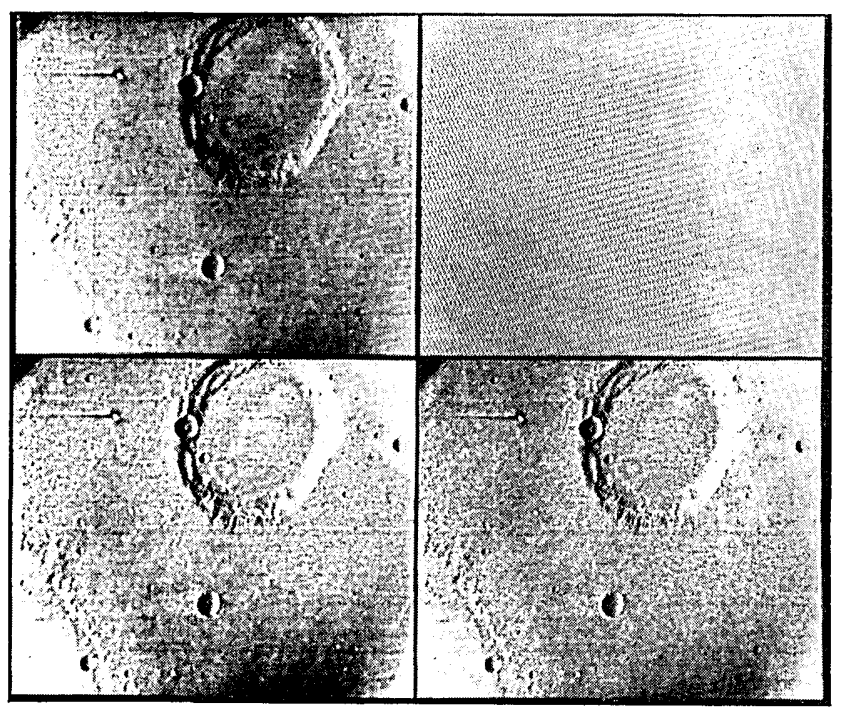

Fig. 4. Processing of Mariner 6 television picture. Upper left: raw analog data of near encounter frame 18 from Mariner 6 as received in August 1969, including on-board enhancement effects. Upper right: electrical noise to be removed from raw data. Lower left: raw data with noise removed and then enhanced, but without correction for vidicon response. Lower right: same, except processed with high frequency filter. All these processing steps were carried out within a few days of receipt of

data. Further information can be found in Refs. 5 and 6 . 
meter at a specified contrast. Contrast is used here specifically to mean the ratio of the maximum intensity to the minimum across a bar chart, or across a sinusoidal variation pattern.

Returning to our discussion of a pixel, there must be a connection between the area of a pixel, with its associated intensity uncertainty, and the limiting resolution vs contrast relationship. Since the output signal-tonoise from the sensor depends on the actual area involved, certainly a high contrast image pattern can be recognized under conditions of worse signal-to-noise than can a low contrast pattern. Therefore, we should expect on very general grounds a relationship between resolution and contrast qualitatively similar to that observed. Conversely, we can sharpen up our concept of a pixel by identifying its area with a resolution element corresponding to a particular contrast, and then choosing a value of $\mathrm{S} / \mathrm{N}$ appropriate for the same contrast. Yet again we find ourselves dealing with intrinsically imprecise concepts, because resolution ultimately depends on the threshold of eye-brain recognition of a standard pattern. It is not possible formally to relate the physically meaningful term signal-to-noise to what is basically a psychophysical process. Thus, again we will have to fall back upon engineering experience and use what empirical information may be available.

For this purpose we define $R$ in line pairs per millimeter as the limiting resolution of an imaging system determined by the visual viewing of a series of three bar targets to determine the most closely spaced detectable pattern. $R$ is obviously a function of the contrast of the pattern. We then imagine that the same image used to determine $R$ is scanned with a (noiseless) microphotometer whose slit width just equals $R / 2$. Under those conditions, the observed signal-to-noise, denoted $q$, is the empirical quantity of interest to us here. The signal-to-noise ratio $q$ has, in fact, been measured for the Lunar Orbiter film system and "SNR's considerably in excess of one were nevertheless obtained." A value of $q$ of about 1.4 is indicated by Fig. 5 of that paper. Brock (Ref. 7, p. 65), on the other hand, comments that "... the modulation of density across the target image is found to be approximately equal to the granularity calculated for an aperture having the area of one bar of the target. Thus the signal-to-noise ratio is on the order of one." Finally, Fulton, CBS Labs (personal communication) finds $q$ to be about 2.5 in an unpublished survey. Thus $q$ seemingly lies between 1 and 2.5. We will use the value of 1.5 here, presuming that the uncertainty in its use does not exceed a factor of two. Therefore,

$$
V_{S} / V_{N_{R}}=q
$$

But what is the corresponding input $\mathrm{S} / \mathrm{N}$ ? We know that the observed input signal corresponded to a particular contrast $c$. If we represent the responsivity $\rho$ and the appropriate MTF corresponding to the limiting resolution $R$ as $Z(R \rho)$, then
$V_{S_{R}}=\left(i_{\max }-i_{\min }\right) Z(R \rho)=\left(i_{\max }+i_{\min }\right) \frac{i_{\max }-i_{\min }}{i_{\max }+i_{\min }} Z(R \rho)$

Since $i_{\max }+i_{\min }=2 i$,

$$
V_{S_{R}}=2 i[(c-1) /(c+1)] Z(R \rho)
$$

and

$$
V_{N R}=\delta i Z(R \rho) .
$$

Therefore,

$$
i / \delta i_{R}=(q / 2)[(c+1) /(c-1)] .
$$

\section{The Fundamental Significance of a priori Knowledge}

So far, we have followed the notion of a photograph as a link in a communication system which relays the "view from space" to the ground-based observer as faithfully as possible, or with intentional enhancement in some cases. Yet, the ultimate purpose of all this is to convey information* to the photo interpreter. $\mathrm{He}$ must recognize familiar features and detect and investigate unfamiliar ones. Thus it is necessary to relate the technical parameters of resolution and total number of bits to the more subjective notion of interpretability. The key to this relationship is the extent to which prior knowledge of, or familiarity with, the surface features included in the scene is available. In fact, the basic relationship can be stated as follows: The interpretability of the physical features displayed in a photograph depends on (1) the total information content, and (2) the observer's familiarity with or prior knowledge of those surface features revealed at the surface resolution of that photograph. There is no independent significance to ground resolution per se. ${ }^{9}$ Thus in the early phases of planetary exploration, ground resolution may be regarded as a dependent variable, to be adjusted in increasing steps from a scale comparable to prior photography down to some limit determined by the total information capability of the mission as well as by technical limitations to high resolution photography.

We are led to conclude, therefore, that any space photography endeavor must be conceived, carried out, and ultimately interpreted in terms of what a priori information also exists, especially photographic. The information actually communicated by means of a space photography system depends entirely on how many new features can be recognized by the interpreter. His capability to recognize new features depends on his a priori knowledge concerning similar or related fea-

* We have tried to distinguish between communicated data with which to reconstruct photographs, measured in units of bits, and communicated information which also can be measured in bits. A photograph which is entirely incomprehensible to an observer because of his lack of familiarity with the scene viewed contains no information, regardless of how many bits were required to communicate it. 
tures. Thus the amount and nature of a priori knowledge should be the principal guide as to how the communicated bits are to be expended, especially regarding the balancing of geographic coverage vs ground resolution within whatever total information return limitation is placed by spacecraft and mission considerations.

\section{A Figure of Merit Concerning Resolution}

After the above tradeoffs have been considered, the question of achievable ground resolution becomes the principal basis for the evaluation of spacecraft camera alternatives. However, performance of television systems is often specified in terms of signal-to-noise per TV line and an MTF curve, while that of photographic systems is often specified in terms of limiting bar chart resolution at some specified contrast. Furthermore, photographs taken from the earth, which necessarily supply the a priori background for early planetary missions, are normally evaluated in terms of the associated seeing disk or assigned a limiting ground resolution subjectively by an astronomer. How can this variety of imagery be placed on a common basis as far as ground resolution is concerned? Our proposal is simple: the most unbiased basis for evaluation of ground resolution is the relative amount of photographic information returned per unit surface area of the planet. And the information content of the various kinds of imagery can be estimated from the number of bits per unit surface area required to encode for transmission, without significant degradation, the scene intensities reconstructed from each kind of imagery. Specifically, we define the figure of merit for resolution, FOM, as the number of bits required to encode without significant degradation the intensity reconstructed from an image by a particular system of a unit surface area of the target expressed in square kilometers.

Equation (6) forms the basis of expressing the FOM for a TV system, except that we must relate flat field (uniform input intensity) signal-to-noise to a $\mathrm{S} / \mathrm{N}$ which refers to some spatially varying intensity. This relationship can be obtained through (1) the system MTF curve, which expresses the appropriate intensity attenuation factor as a function of TV lines, and (2) the assumption that the noise among adjacent TV lines is uncorrelated. Under these circumstances, the equivalent input signal-to-noise, $i / \delta i$, is related to the flat field output $\mathrm{S} / \mathrm{N}$,

$$
i / \delta i=(\mathrm{MTF})\left(K_{0} / K_{\mathrm{MTFF}}\right)(\mathrm{S} / \mathrm{N})_{F}+1,
$$

where MTF equals the attenuation coefficient for a particular space frequency, $K_{\mathrm{ATF}}$ is TV lines $/ \mathrm{mm}$ corresponding to that $\mathrm{MTF}$, and $K_{0}$ is the TV lines/ $\mathrm{mm}$ in the format, and $(\mathrm{S} / \mathrm{N})_{F}$ is the flat field signal-tonoise.

At this point, we come to the same kind of arbitrariness encountered with film systems when it is necessary to specify a particular contrast at which limiting resolution will be specified. In both cases, some form of weighted integral would seem indicated. Yet in both cases the relevant test data for such integrals are rarely available until after system choices have been made. Also, the use of integrals implies some kind of a priori weighting of the expected spatial and contrast content of the unknown scene and thus a bias as to the results. Hence, it is desirable in practice to choose a nominal MTF for evaluation of TV systems, recognizing some oversimplification is necessarily involved. In the present case we choose the MTF value of 0.2 as the highest spatial resolution point on the MTF curve which can be reliably estimated in advance from design data. Accordingly

$$
i / \delta i=0.2\left(K_{0} / K_{0.2}\right)(\mathrm{S} / \mathrm{N})_{F}+1
$$

and

$\mathrm{FOM}_{\mathrm{TV}}=\left(3 Y f^{2} K_{0.2^{2}} / H^{2}\right) \log _{10}\left[1+\left(0.2 K_{0} / K_{0.2}\right)(\mathrm{S} / \mathrm{N})_{F}\right]$,

where $f$ is the effective focal length in millimeters and $H$ the range in kilometers to the target.

Similarly, Eq. (20) yields

$\mathrm{FOM}_{\mathrm{fi} 1 \mathrm{~m}}=\left(12 R^{2} Y f^{2} / H^{2}\right) \log _{10}\{1+[q(c+1) / 2(c-1)]\}$.

And, if we denote $S$ to be the minimum detectable dimension of a surface feature of intrinsic contrast $C$ in a ground-based planetary photograph, ${ }^{*}$

$$
\mathrm{FOM}_{\mathrm{GB}}=\left(12 Y / S^{2}\right) \log _{10}\{1+[q(c+1) / 2(c-1)]\} .
$$

As an example, we will compare the number of bits per unit surface area which could be returned from a high resolution Lunar Orbiter camera system hypothetically placed in orbit about Mars with the number to be returned from the Mariner TV camera system to be deployed in 1971.

Combining Eqs. (23) and (24), and assuming both systems operate from a $1500-\mathrm{km}$ closest approach,

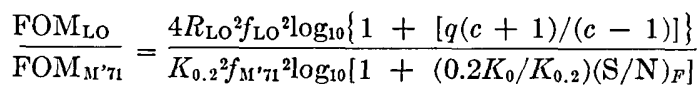

and, noting from Table III and Fig. 2, that $(\mathrm{S} / \mathrm{N})_{F}=$ $75, f_{\mathrm{LO}}=610 \mathrm{~mm}, f_{\mathrm{II}^{\prime} 71}=508 \mathrm{~mm}, K_{0.2}=30 \mathrm{~mm}^{-1}$, $R_{\mathrm{LO}}=761_{p} / \mathrm{mm}$ at contrast of $3: 1, K_{0}=74 \mathrm{~mm}^{-1}$, and $q=1.5$ then,

$$
\frac{\mathrm{FOM}_{\mathrm{LO}}}{\mathrm{FOM}_{\mathrm{AI}}{ }^{2} 71}=\frac{4(76)^{2}(610)^{2} \log _{10}(1+1.5)}{(30)^{2}(508)^{2} \log _{10}[1+(74 / 30)(75 / 5)]}=9.2 .
$$

Thus, even at a range of $1500 \mathrm{~km}$, a Lunar Orbiter system would appear to yield an order of magnitude greater information per unit surface area than will the kind of system to be flown around Mars by the U.S. in

*Inasmuch as the minimum detectable pattern in such a case is not a repetitious one, the appropriate value of $q$ may be higher than for the film tests discussed earlier. However, we will continue to use the value 1.5 in the absence of published data to the contrary. 
Table III. Comparison of Mariner Mars 1971 and Lunar Orbiter ${ }^{a}$ Camera Systems

\begin{tabular}{|c|c|c|c|c|}
\hline & \multicolumn{2}{|c|}{ Lunar orbiter } & \multicolumn{2}{|c|}{ Mariner Mars '71 } \\
\hline System weight & \multirow{2}{*}{\multicolumn{2}{|c|}{$\begin{array}{c}147 \mathrm{lb} \\
\text { two frames }\end{array}$}} & \multicolumn{2}{|c|}{$68.3 \mathrm{lb}$} \\
\hline Camera types & & & \multicolumn{2}{|c|}{ two frames } \\
\hline Sensor & \multicolumn{2}{|c|}{ photographic films } & \multicolumn{2}{|c|}{ two vidicons } \\
\hline \multirow{2}{*}{$\begin{array}{l}\text { Lens focal length } \\
\text { focal ratio }\end{array}$} & $80 \mathrm{~mm}$ & $610 \mathrm{~mm}$ & $50 \mathrm{~mm}$ & $508 \mathrm{~mm}$ \\
\hline & $f / 5.6$ & $f / 5.6$ & $f / 4$ & $f / 2.5$ \\
\hline \multirow[t]{2}{*}{ Format } & $38^{\circ} \times 44^{\circ}$ & $5.2^{\circ} \times 20.4^{\circ}$ & $11^{\circ} \times 14^{\circ}$ & $1.1^{\circ} \times 1.4^{\circ}$ \\
\hline & $55 \mathrm{~mm} \times 65 \mathrm{~mm}$ & $55 \mathrm{~mm} \times 219 \mathrm{~mm}$ & $9.6 \mathrm{~mm} \times 12.5 \mathrm{~mm}$ & $9.6 \mathrm{~mm} \times 12.5 \mathrm{~mm}$ \\
\hline Readout pixels/line & 9,880 & 33,288 & 832 & 832 \\
\hline lines/frame & 8,360 & 8,360 & 700 & 700 \\
\hline Pixels per frame & $8.3 \times 10^{7}$ & $2.8 \times 10^{8}$ & $5.8 \times 10^{5}$ & $5.8 \times 10^{5}$ \\
\hline $\begin{array}{l}\text { System resolution } \\
\text { parameters }\end{array}$ & \multicolumn{2}{|c|}{$\begin{array}{l}\text { limiting resolution of } 76 \mathrm{lp} / \mathrm{mm} \\
\text { at } 3: 1 \text { contrast and } \mathrm{S} / \mathrm{N}=1.5\end{array}$} & \multicolumn{2}{|c|}{$\begin{array}{l}\text { flat field signal-to-noise } \sim 75 / 1 \\
20 \% \text { response at } 30 \mathrm{TV} \text { lines } / \mathrm{mm}\end{array}$} \\
\hline \multicolumn{5}{|l|}{$\begin{array}{l}\text { Projected frame size } \\
\text { (from } 1,500 \mathrm{~km} \text { ) }\end{array}$} \\
\hline along flight & $1,035 \mathrm{~km}$ & $135 \mathrm{~km}$ & $285 \mathrm{~km}$ & $28.5 \mathrm{~km}$ \\
\hline cross flight & $1,215 \mathrm{~km}$ & $540 \mathrm{~km}$ & $375 \mathrm{~km}$ & $37.5 \mathrm{~km}$ \\
\hline \multicolumn{5}{|l|}{ Projected pixel size } \\
\hline (from $1,500 \mathrm{~km}$ ) & $108 \mathrm{~m}$ & $16 \mathrm{~m}$ & $420 \mathrm{~m}$ & $42 \mathrm{~m}$ \\
\hline Periapsis smear at & \multirow{2}{*}{\multicolumn{2}{|c|}{ compensated }} & $21 \mathrm{~m}$ at $6 \mathrm{msec}$ & $10 \mathrm{~m}$ at $3 \mathrm{msec}$ \\
\hline $3.5 \mathrm{~km} / \mathrm{sec}$ & & & $168 \mathrm{~m}$ at $48 \mathrm{msec}$ & $89 \mathrm{~m}$ at $24 \mathrm{msec}$ \\
\hline
\end{tabular}

a The pixel size and line widths shown here refer to the design performance of $76 \mathrm{lp} / \mathrm{mm}$ and 152 scan lines/mm. The actual lunar orbiter system used $278 \mathrm{scan}$ lines $/ \mathrm{mm}$ and is reported to have exceeded $100 \mathrm{lp} / \mathrm{mm}$ at $3: 1$ contrast.

1971.* Furthermore, the Lunar Orbiter type system has a built-in image motion compensation system (which also could be provided for a TV system) permitting it to be operated much closer to the surface than can the Mariner ' 71 system which is virtually smear limited to $1500 \mathrm{~km}$. Hence, it is clear that the basic space-proven technology already exists which could make possible a dramatic breakthrough in Mars surface resolution in, say, 1973, assuming the weight, power, and cost increments were deemed to be warranted.

Another kind of comparison which must be carried out frequently involves the comparison of space probe photography with good ground-based planetary photographs. We ask the very real operational question: At what distance away from Mars would spacecraft photography of the whole disk have an information content approximately equal to that of the very best ground-based photographs? $\dagger$ Assuming the spacecraft to be of the Mariner Mars '69 or '71 type, and equating Eqs. (24) and (25),

\footnotetext{
* Keene, Eastman-Kodak (private communication) indicates that actual flight performance of the Lunar Orbiter system was higher than the test value of $76 \mathrm{Ip} / \mathrm{mm}$ quoted here, and, in fact, gave at least $100 \mathrm{lp} / \mathrm{mm}$ at $3: 1$ contrast. Use of this performance number would increase the apparent advantage over the television system from 9.2 to 13.8 .

$\dagger$ It would be desirable to begin even earlier in order to have the benefit of continuous, uniform coverage.
}

$$
\begin{aligned}
\frac{3 Y f^{2} K^{2}{ }_{0.2}}{H^{2}} \log _{10}\left[1+\frac{0.2 K_{0}}{K_{0.2}}\right. & \left.\left(\frac{\mathrm{S}}{\mathrm{N}}\right)_{F}\right] \\
& =\frac{12 Y}{S^{2}} \log _{10}\left[1+\frac{q(c+1)}{2(c-1)}\right]
\end{aligned}
$$

and

$$
H^{2}=\frac{S^{2} f^{2} K_{0.2}^{2}}{4} \frac{\log _{10}\left[1+\left(0.2 K_{0} / K_{0.2}\right)(\mathrm{S} / \mathrm{N})_{F}\right]}{\log _{10}\{1+[q(c+1) / 2(c-1)]\}} .
$$

Taking the very best ground-based resolution to be $100 \mathrm{~km}$ for a feature of $2: 1$ contrast, and using the previous values for the ' 71 system, we find $H=1.3 \times 10^{6}$ $\mathrm{km}$. This corresponds to a little more than two days before encounter in the case of 1969 or 1971 and is in approximate agreement with the actual results from the far encounter pictures acquired by Mariner 6 and 7. Analogous calculations from Jupiter and Mercury for the same kind of camera system indicate equivalent information content at about 17 days $\left(12 \times 10^{6} \mathrm{~km}\right)$ out from Jupiter and at a little more than two days $\left(2.2 \times 10^{6} \mathrm{~km}\right)$ from Mercury.*

The FOM we have discussed in this section is based solely on relative surface information communicated;

\footnotetext{
* Assuming a best ground-based resolution of $250 \mathrm{~km}$ at $10 \%$ contrast for Mercury and an average night-to-night ground-based resolution of $1200 \mathrm{~km}$ at $20 \%$ contrast for Jupiter, the appropriate value for a temporally varying object. We are indebted to B. A. Smith of New Mexico State University Observatory for these numbers. Encounters in 1973 were assumed with approach speeds around $13 \mathrm{~km} / \mathrm{sec}$ for Mercury and $8 \mathrm{~km} / \mathrm{sec}$ for Jupiter.
} 
it does not distinguish between high signal-to-noise (and large pixels) and lower signal-to-noise (and smaller pixels). Should a priori information or attitudes be available such that the desired signal-to-noise per pixel could be stated in advance, then a more responsive FOM could be developed based upon the relative surface area imaged by each system at the specified signalto-noise. This procedure affords a way of taking into account more fully the differing MTF curves and noise distributions of various candidate systems.

\section{Where Next?}

Mariner 4 provided man's first close-up look at Mars by furnishing $3.75 \times 10^{6}$ bits of photographic data obtained from less than $1 \%$ of the surface under somewhat unfavorable operational conditions. Yet, even so, a strong morphological resemblance to the lunar surface was discovered, a circumstance which had not been generally anticipated.

Mariners 6 and 7 have just flown by Mars, one on an equatorial and one on a polar trajectory, and have returned over one hundred times the picture data of Mariner 4. These data bits contain relatively more information because the photographic experience from Mariner 4 has been incorporated into their design by maximizing the return of low contrast scene information. Mariner '69 was indeed the first systematic exploration of the planet in that major elements of the surface were sampled including light and dark areas, a bright desert, the polar cap, and others. It also provided low resolution coverage of the entire planet (by use of the high resolution camera before encounter), Mariner-4-type resolution along carefully selected paths for about $20 \%$ of the planet, and nested high resolution samples down to several hundred meters ground resolution.

The 1971 Mars Orbiter mission should permit the return of an additional factor of $10^{2}$ bits over the 1969 mission or a factor of more than $10^{4}$ over Mariner 4. Thus, there will be the opportunity for systematic mapping of fixed features and the monitoring of variable features.

However, the 1971 mission faces two very significant constraints. First, it was required to use much of the hardware of the ' 69 mission in a manner for which it was not designed. Specifically, the camera and data systems must be operated for several hundred operational cycles instead of merely one or two. There also will be the need and desire to revise operational parameters and procedures during the mission operation. Hence real questions of reliability and efficiency are involved.

Secondly, most of the redesign of the '69 hardware for the '71 mission had to be carried out before the scientific and performance results of the '69 mission were obtained. Yet the '71 mission must incorporate essential information from ' 69 on optimum lighting and any latitudinal dependence, on relative visibility with different filters, and on the appearance and possible location of new, unexpected features. Fortunately, the initial results of Mariner 6 and 7 do not seem to indicate major changes in hardware for the '71 mission. However, scientific priorities and nominal mission profiles may well warrant reconsideration in view of the variety of terrain, the paucity of clouds, and the diversity of transitory features observed at the edge and on the polar cap.

Initial considerations of the orbit suggested that it is preferable to allocate one of the spacecraft in 1971 to the mapping of fixed features and the other to monitor variable features. For the mapping of fixed topographic features, systematic photography of successive portions of the Martian surface from about the same altitude and viewing aspect, and especially at the same, probably low-lighting, angle is essential. Study of the wave of darkening, local frost deposits, the polar cap, and cloud phenomena, on the other hand, require frequent return to the same area, presumably at the expense of total coverage. In addition, higher lighting angles may be desirable in order to minimize topographic and maximize albedo contributions to the observed terrain brightness.

The first lander, Viking, is planned for 1973, at which time pictures from the orbiting bus can be obtained, particularly in the area of the lander, at much better resolution than previously possible. These pictures will be needed to orient and correlate the lander information, to understand the finer structure of the planetary surface, and to classify the surface area in which the lander arrived. However, a further order of magnitude increase in surface resolution cannot be obtained with the type of photographic systems used so far in the Mariner program, although the basic space technology needed for several orders of magnitude increase exists. Thus, a new camera design is indicated for the '73 mission which will be capable of a sharp increase in surface resolution; then it will represent as significant a step in Martian exploration as have previous U.S. photographic missions.

Mars will be the first planet, aside from the earth, to be explored remotely from space. As pointed out previously, ${ }^{8}$ this exciting series of projects should not be initiated to the exclusion of all other planetary missions in the early '70's. Certain flyby missions which use gravity assist are very cost effective and permit first looks at a number of planets. The 1973 Venus/Mercury mission is of this type, as are the flights to the outer planets in the later '70's. The Jupiter/Saturn/Pluto and Jupiter/Uranus/Neptune tours will take from seven to nine years and in most cases will terminate only with loss of communication with the spacecraft as it leaves the solar system. The next decade, man willing, should go down in history as the flowering period for planetary exploration, as favorable conditions for such economical (gravity assist) missions will not be available again for many decades. Space photography will be a principal exploratory tool, and, as well, will permit mankind generally to share in this exciting visual process.

Parts of the material presented here are condensed from the draft manuscript of a book prepared under 
RAND Corporation sponsorship entitled, The View from Space: Space Exploration by Photography. The Figure of Merit discussion was developed by B. Murray under support to Caltech from Grant NGR-05-002-117 of the National Aeronautics and Space Administration.

We are pleased also to acknowledge the helpful criticism of Horace Ory of RAND, Robert Leighton of Caltech, James Fulton of CBS Labs, and George Keene of Eastman Kodak.

This paper is Contribution 1642 of the Division of Geological Sciences of the California Institute of Technology and P-4120 of the RAND Corporation.

\section{References}

1. D. Smith, Appl. Opt. 2, 335 (1963).

2. G. C. Brock, in Photographic Considerations for Aerospace (Itek Corporation, Lexington, Mass., 1965), Chap. 3, pp. 4272 .

3. R. B. Leighton, B. C. Murray, R. P. Sharp, J. D. Allen, and R. K. Sloan, JPL Tech. Rept. 32-884, 15 December 1967.

4. B. L. Elle, C. S. Hernmiller, P. J. Fromme, and A. E. Neumer, J. Soc. Motion Picture Television Engrs. 76, 733 (1967).

5. R. B. Leighton, N. H. Horowitz, B. C. Murray, R. P. Sharp, A. G. Herriman, A. T. Young, B. A. Smith, M. E. Davies, and C. B. Leovy, Science 165, 684 (1969).

6. R. B. Leighton, N. H. Horowitz, B. C. Murray, R. P. Sharp, A. G. Herriman, A. T. Young, B. A. Smith, M. E. Davies, and C. B. Leovy, Science 165, 787 (1969).

7. G. C. Brock, in Useful Applications of Earth-Oriented Satellites (Natl. Acad. Sciences, Wash. D. C., 1969), 6, p. 63.

8. B. C. Murray, Astron. Aeron. 6, No. 10, 42 (1968).

9. B. C. Murray, Advan. Astron. Sci. 19, 153 (1966).

\section{CALL FOR PAPERS}

A Special Issue of the Proceedings of the IEEE devoted to Thick and Thin Film Technologies for Microelectronic (chip and substrate), Microwave, Memory, and Optoelectronic Applications is planned for publication in September 1971. Papers dealing with the preparation, evaluation, and application of films in these categories are sought. Authors wishing to submit papers for consideration are requested to provide detailed, unambiguous abstracts not exceeding 250 words in length, on or before 15 October 1970. These should be followed by complete papers, to be received on or before 15 January 1971 . Guest co-editors for this issue to whom papers are to be submitted:

A. Reisman

IBM Thomas J. Watson Research Center

P.O. Box 218

Yorktown Heights, N.Y. 10598
K. Rose

Electrophysics Division

Rensselaer Polytechnic Institute

Troy, N.Y. 12181 